\title{
Some Approximation Properties of Modified Jain-Beta Operators
}

\author{
Vishnu Narayan Mishra ${ }^{1,2}$ and Prashantkumar Patel ${ }^{1,3}$ \\ ${ }^{1}$ Department of Applied Mathematics \& Humanities, Sardar Vallabhbhai National Institute of Technology, \\ Ichchanath Mahadev Road, Surat, Gujarat 395 007, India \\ ${ }^{2}$ L. 1627 Awadh Puri Colony Beniganj, Phase-III, Opp. I.T.I., Ayodhya Main Road Faizabad, Uttar Pradesh 224 001, India \\ ${ }^{3}$ Department of Mathematics, St. Xavier College, Ahmedabad, Gujarat 380 009, India
}

Correspondence should be addressed to Vishnu Narayan Mishra; vishnunarayanmishra@gmail.com

Received 21 May 2013; Revised 24 October 2013; Accepted 3 December 2013

Academic Editor: Jacob Engwerda

Copyright (C) 2013 V. N. Mishra and P. Patel. This is an open access article distributed under the Creative Commons Attribution License, which permits unrestricted use, distribution, and reproduction in any medium, provided the original work is properly cited.

Generalization of Szász-Mirakyan operators has been considered by Jain, 1972. Using these generalized operators, we introduce new sequences of positive linear operators which are the integral modification of the Jain operators having weight functions of some Beta basis function. Approximation properties, the rate of convergence, weighted approximation theorem, and better approximation are investigated for these new operators. At the end, we generalize Jain-Beta operator with three parameters $\alpha, \beta$, and $\gamma$ and discuss Voronovskaja asymptotic formula.

\section{Introduction}

For $0<\vartheta<\infty,|\kappa|<1$, let

$$
w_{\kappa}(i, \vartheta)=\vartheta(\vartheta+i \kappa)^{i-1} \frac{e^{-(\vartheta+i \kappa)}}{i !} \quad i=0,1,2, \ldots ;
$$

then

$$
\sum_{i=0}^{\infty} w_{\kappa}(i, \vartheta)=1
$$

Equation (1) is a Poisson-type distribution which has been considered by Consul and Jain [1].

In 1970, Jain [2] introduced and studied the following class of positive linear operators:

$$
P_{n}^{\kappa}(f, x)=\sum_{i=0}^{\infty} w_{\kappa}(i, n x) f\left(\frac{i}{n}\right),
$$

where $0 \leq \kappa<1$ and $w_{\kappa}(i, n x)$ has been defined in (1).

The parameter $\kappa$ may depend on the natural number $n$. It is easy to see that $\kappa=0 ;(3)$ reduces to the well-known
Szász-Mirakyan operators [3]. Different generalization of Szász-Mirakyan operator and its approximation properties is studied in $[4,5]$. Kantorovich-type extension of $P_{n}^{\kappa}$ was given in [6]. Integral version of Jain operators using Beta basis function is introduced by Tarabie [7], which is as follows:

$$
\begin{aligned}
P_{n}^{\kappa}(f, x)= & \sum_{i=1}^{\infty} \frac{1}{B(n+1, i)} w_{\kappa}(i, n x) \\
& \times \int_{0}^{\infty} \frac{t^{i-1}}{(1+t)^{n+i+1}} f(t) d t+e^{-n x} f(0) .
\end{aligned}
$$

In Gupta et al. [8] they considered integral modification of the Szász-Mirakyan operators by considering the weight function of Beta basis functions. Recently, Dubey and Jain [9] considered a parameter $\gamma$ in the definition of [8]. Motivated by such types of operators we introduce new sequence of linear operators as follows:

$$
\text { For } x \in[0, \infty) \text { and } \gamma>0 \text {, }
$$

$$
P_{n, \gamma}^{\kappa}(f, x)=\sum_{i=1}^{\infty} w_{\kappa}(i, n x) \int_{0}^{\infty} b_{n, i, \gamma}(t) f(t) d t+e^{-n x} f(0),
$$


where $w_{\kappa}(i, n x)$ is defined in (1) and

$$
b_{n, i, \gamma}(t)=\frac{\gamma \Gamma(n / \gamma+i+1)}{\Gamma(i) \Gamma(n / \gamma+1)} \frac{(\gamma t)^{i-1}}{(1+\gamma t)^{(n / \gamma)+i+1}}
$$

The operators defined by (5) are the integral modification of the Jain operators having weight function of some Beta basis function. As special case, $\gamma=1$ the operators (5) reduced to the operators recently studied in [7]. Also, if $\kappa=0$ and $\gamma=1$, then the operators (5) turn into the operators studied in [8].

In the present paper, we introduce the operators (5) and estimate moments for these operators. Also, we study local approximation theorem, rate of convergence, weighted approximation theorem, and better approximation for the operators $P_{n, \gamma}^{\kappa}$. At the end, we propose Stancu-type generalization of (5) and discuss some local approximation properties and asymptotic formula for Stancu-type generalization of Jain-Beta operators.

\section{Basic Results}

Lemma 1 (see [2]). For $P_{n}^{\kappa}\left(t^{m}, x\right), m=0,1,2$, one has

$$
\begin{gathered}
P_{n}^{\kappa}(1, x)=1, \quad P_{n}^{\kappa}(t, x)=\frac{x}{1-\kappa}, \\
P_{n}^{\kappa}\left(t^{2}, x\right)=\frac{x^{2}}{(1-\kappa)^{2}}+\frac{x}{n(1-\kappa)^{3}} .
\end{gathered}
$$

Lemma 2. The operators $P_{n, \gamma}^{\kappa}, n>\gamma$ defined by (5) satisfy the following relations:

$$
\begin{gathered}
P_{n, \gamma}^{\kappa}(1, x)=1, \quad P_{n, \gamma}^{\kappa}(t, x)=\frac{x}{1-\kappa}, \\
P_{n, \gamma}^{\kappa}\left(t^{2}, x\right)=\frac{n x^{2}}{(1-\kappa)^{2}(n-\gamma)}+\frac{x\left(1+(1-\kappa)^{2}\right)}{(1-\kappa)^{3}(n-\gamma)} .
\end{gathered}
$$

Proof. By simple computation, we get

$$
\begin{aligned}
P_{n, \gamma}^{\kappa}(1, x)= & \sum_{i=1}^{\infty} w_{\kappa}(i, n x)+e^{-n x}=P_{n}^{\kappa}(1, x)=1 \\
P_{n, \gamma}^{\kappa}(t, x)= & \sum_{i=1}^{\infty} w_{\kappa}(i, n x) \\
& \times \int_{0}^{\infty} \frac{\gamma \Gamma(n / \gamma+i+1)}{\Gamma(i) \Gamma(n / \gamma+1)} \cdot \frac{(\gamma t)^{i-1}}{(1+\gamma t)^{(n / \gamma)+i+1}} \cdot t d t \\
= & \sum_{i=1}^{\infty} w_{\kappa}(i, n x) \frac{\gamma \Gamma(n / \gamma+i+1)}{\Gamma(i) \Gamma(n / \gamma+1)} \\
= & \sum_{i=1}^{\infty} w_{\kappa}(i, n x) \frac{\Gamma(i+1) \Gamma(n / \gamma)}{\gamma^{2} \Gamma(n / \gamma+i+1)}=P_{n}^{\kappa}(t, x)=\frac{x}{1-\kappa}
\end{aligned}
$$

$$
\begin{aligned}
& P_{n, \gamma}^{\kappa}\left(t^{2}, x\right)=\sum_{i=1}^{\infty} w_{\kappa}(i, n x) \\
& \times \int_{0}^{\infty} \frac{\gamma \Gamma(n / \gamma+i+1)}{\Gamma(i) \Gamma(n / \gamma+1)} \cdot \frac{(\gamma t)^{i-1}}{(1+\gamma t)^{(n / \gamma)+i+1}} \cdot t^{2} d t \\
& =\sum_{i=1}^{\infty} w_{\kappa}(i, n x) \frac{\gamma \Gamma(n / \gamma+i+1)}{\Gamma(i) \Gamma(n / \gamma+1)} \\
& \frac{\Gamma(i+2) \Gamma((n / \gamma)-1)}{\gamma^{3} \Gamma(n / \gamma+i+1)} \\
& =\sum_{i=1}^{\infty} w_{\kappa}(i, n x) \frac{i^{2}+i}{n(n-\gamma)} \\
& =\sum_{i=1}^{\infty} w_{\kappa}(i, n x)\left(\frac{i^{2}}{n(n-\gamma)}+\frac{i}{n(n-\gamma)}\right) \\
& =\frac{n}{n-\gamma} \sum_{i=1}^{\infty} w_{\kappa}(i, n x) \frac{i^{2}}{n^{2}}+\frac{1}{n-\gamma} \sum_{i=1}^{\infty} w_{\kappa}(i, n x) \frac{i}{n} \\
& =\frac{n}{n-\gamma}\left(\frac{x^{2}}{(1-\kappa)^{2}}+\frac{x}{n(1-\kappa)^{3}}\right) \\
& +\frac{1}{(n-\gamma)} \frac{x}{(1-\kappa)} \\
& =\frac{n x^{2}}{(1-\kappa)^{2}(n-\gamma)}+\frac{x\left(1+(1-\kappa)^{2}\right)}{(1-\kappa)^{3}(n-\gamma)} \text {. }
\end{aligned}
$$

Lemma 3. For $x \in[0, \infty), n>\gamma$, and with $\varphi_{x}=t-x$, one has

$$
\begin{aligned}
& \text { (i) } P_{n, \gamma}^{\kappa}\left(\varphi_{x}, x\right)=\kappa x /(1-\kappa), \\
& \text { (ii) } P_{n, \gamma}^{\kappa}\left(\varphi_{x}^{2}, x\right)=\left(\left(\kappa^{2}(n-\gamma)+\gamma\right) /\left((1-\kappa)^{2}(n-\gamma)\right)\right) x^{2}+ \\
& \quad\left(\left(1+(1-\kappa)^{2}\right) /\left((1-\kappa)^{3}(n-\gamma)\right)\right) x
\end{aligned}
$$

Lemma 4. For $x \in[0, \infty), n>\gamma$, one has

$$
P_{n, \gamma}^{\kappa}\left(\varphi_{x}^{2}, x\right) \leq \frac{n+2}{(1-\kappa)^{3}(n-\gamma)}\left(x^{2}+x\right)=\delta_{\kappa, n, \gamma}(x) \quad(\text { say }) .
$$

Proof. Since $\max \left\{x, x^{2}\right\} \leq x+x^{2},(1-\kappa)^{2} \leq 1$, and $(1-\kappa)^{-2} \leq$ $(1-\kappa)^{-3}$, we have

$$
\begin{aligned}
P_{n, \gamma}^{\kappa}\left(\varphi_{x}^{2}, x\right) & \leq\left[\frac{\kappa^{2}(n-\gamma)+\gamma+2}{(1-\kappa)^{3}(n-\gamma)}\right]\left(x+x^{2}\right) \\
& \leq\left[\frac{n+2}{(1-\kappa)^{3}(n-\gamma)}\right]\left(x+x^{2}\right) \quad(\text { as }|\kappa|<1),
\end{aligned}
$$

which is required. 


\section{Some Local Approximation}

Let $B_{x^{2}}[0, \infty)=\left\{f\right.$ :for every $x \in[0, \infty),|f(x)| \leq M_{f}(1+$ $\left.x^{2}\right), M_{f}$ being a constant depending on $\left.f\right\}$. By $C_{x^{2}}[0, \infty)$, we denote the subspace of all continuous functions belonging to $B_{x^{2}}[0, \infty)$. Also, $C_{x^{2}}^{*}[0, \infty)$ is subspace of all the function $f \in C_{x^{2}}[0, \infty)$ for which $\lim _{x \rightarrow \infty}\left(f(x) /\left(1+x^{2}\right)\right)$ is finite. The norm on $C_{x^{2}}^{*}[0, \infty)$ is $\|f\|_{x^{2}}=\sup _{x \in[0, \infty)}\left(|f(x)| /\left(1+x^{2}\right)\right)$.

If we look at Lemma 2 and based on the famous Korovkin theorem [10], it is clear that $\left\{P_{n, \gamma}^{\kappa}\right\}_{n>\gamma}$ does not form an approximation process. To do this approximation process, we replace constant $\kappa$ by a number $\kappa_{n} \in[0,1)$. If

$$
\lim _{n \rightarrow \infty} \kappa_{n}=0,
$$

then Lemma 2 gives

$$
\lim _{n \rightarrow \infty} P_{n, \gamma}^{\kappa_{n}}\left(t^{j}, x\right)=x^{j}, \quad j=0,1,2,
$$

uniformly on any compact interval $K \subset[0, \infty)$. Based on Korovkin's criteria we state the following.

Theorem 5. Let $P_{n, \gamma}^{\kappa_{n}}$ with $n>\gamma$ be defined in (5), where $\lim _{n \rightarrow \infty} \kappa_{n}=0$. For any compact $K \subset[0, \infty)$ and for each $f \in C_{x^{2}}^{*}[0, \infty)$ one has

$$
\lim _{n \rightarrow \infty} P_{n, \gamma}^{\kappa_{n}}(f, x)=f(x) \text {, uniformly in } x \in K \text {. }
$$

Now, we establish a direct local approximation theorem for the modified operators $P_{n, \gamma}^{\kappa}$ in ordinary approximation. Let the space $C_{B}[0, \infty)$ of all continuous and bounded functions be endowed with the norm $\|f\|=\sup \{|f(x)|: x \in$ $[0, \infty)\}$. Further let us consider the following $K$-functional:

$$
K_{2}(f, \delta)=\inf _{g \in W^{2}}\left\{\|f-g\|+\delta\left\|g^{\prime \prime}\right\|\right\},
$$

where $\delta>0$ and $W^{2}=\left\{g \in C_{B}[0, \infty): g^{\prime}, g^{\prime \prime} \in C_{B}[0, \infty)\right\}$. By the methods given in [11], there exists an absolute constant $C>0$ such that

$$
K_{2}(f, \delta) \leq C \omega_{2}(f, \sqrt{\delta}),
$$

where

$$
\omega_{2}(f, \sqrt{\delta})=\sup _{0<h \leq \sqrt{\delta}} \sup _{x \in[0, \infty)}|f(x+2 h)-2 f(x+h)+f(x)|
$$

is the second order modulus of smoothness of $f \in C_{B}[0, \infty)$.

Theorem 6. For $f \in C_{B}[0, \infty)$ and $n>\gamma$, one has

$$
\begin{aligned}
& \left|P_{n, \gamma}^{\kappa}(f, x)-f(x)\right| \\
& \leq \omega\left(f, \frac{\kappa x}{1-\kappa}\right) \\
& \quad+C \omega_{2}\left(f, \sqrt{\delta_{\kappa, n, \gamma}(x)+\frac{\kappa x}{1-\kappa}}\right),
\end{aligned}
$$

where $C$ is a positive constant.
Proof. We introduce the auxiliary operators as follows:

$$
\widehat{P}_{n, \gamma}^{\kappa}(f, x)=P_{n, \gamma}^{\kappa}(f, x)-f\left(x+\frac{\kappa x}{1-\kappa}\right)+f(x) .
$$

Let $g \in W_{\infty}^{2}$ and $x, t \in[0, \infty)$. By Taylor's expansion we have

$$
g(t)=g(x)+(t-x) g^{\prime}(x)+\int_{x}^{t}(t-u) g^{\prime \prime}(u) d u .
$$

Applying $\widehat{P}_{n, \gamma}^{\kappa}$, we get

$$
\begin{aligned}
\widehat{P}_{n, \gamma}^{\kappa}(g, x)-g(x)= & g^{\prime}(x) \widehat{P}_{n, \gamma}^{\kappa}((t-x), x) \\
& +\widehat{P}_{n, \gamma}^{\kappa}\left(\int_{x}^{t}(t-u) g^{\prime \prime}(u) d u, x\right) .
\end{aligned}
$$

Applying Lemma 2, we get

$$
\begin{aligned}
& \left|\widehat{P}_{n, \gamma}^{\kappa}(g, x)-g(x)\right| \\
& \leq \widehat{P}_{n, \gamma}^{\kappa}\left(\left|\int_{x}^{t}(t-u) g^{\prime \prime}(u) d u\right|, x\right) \\
& \leq P_{n, \gamma}^{\kappa}\left((t-u)^{2}, x\right)\left\|g^{\prime \prime}\right\| \\
& \quad+\left|\int_{x}^{x+\kappa x /(1-\kappa)}\left(x+\frac{\kappa x}{1-\kappa}-u\right) g^{\prime \prime}(u) d u\right| \\
& \leq\left[\delta_{\kappa, n, \gamma}(x)+\frac{\kappa x}{1-\kappa}\right]\left\|g^{\prime \prime}\right\| .
\end{aligned}
$$

Since

$$
\begin{aligned}
\left|P_{n, \gamma}^{\kappa}(f, x)\right| \leq & \sum_{i=1}^{\infty} w_{\kappa}(i, n x) \\
& \times \int_{0}^{\infty} b_{n, i, \gamma}(t)|f(t)| d t+e^{-n x}|f(0)| \leq\|f\|,
\end{aligned}
$$

$$
\begin{aligned}
\left|P_{n, \gamma}^{\kappa}(f, x)-f(x)\right| \\
\leq\left|\widehat{P}_{n, \gamma}^{\kappa}(f-g, x)-(f-g)(x)\right| \\
\quad+\left|\widehat{P}_{n, \gamma}^{\kappa}(g, x)-g(x)\right| \\
\quad+\left|\left(x+\frac{\kappa x}{1-\kappa}\right)-f(x)\right| \\
\leq 2\|f-g\|+\left[\delta_{\kappa, n, \gamma}(x)+\frac{\kappa x}{1-\kappa}\right]\left\|g^{\prime \prime}\right\| \\
\quad+\omega\left(f, \frac{\kappa x}{1-\kappa}\right) .
\end{aligned}
$$

Taking infimum overall $g \in W^{2}$, we get

$$
\begin{aligned}
\left|P_{n, \gamma}^{\kappa}(f, x)-f(x)\right| \leq & K\left(f, \delta_{\kappa, n, \gamma}(x)+\frac{\kappa x}{1-\kappa}\right) \\
& +\omega\left(f, \frac{\kappa x}{1-\kappa}\right) .
\end{aligned}
$$


In view of (18)

$$
\begin{aligned}
& \left|P_{n, \gamma}^{\kappa}(f, x)-f(x)\right| \\
& \quad \leq C \omega_{2}\left(f, \sqrt{\delta_{\kappa, n, \gamma}(x)+\frac{\kappa x}{1-\kappa}}\right)+\omega\left(f, \frac{\kappa x}{1-\kappa}\right),
\end{aligned}
$$

which proves the theorem.

\section{Rate of Convergence and \\ Weighted Approximation}

For any positive $a$, by

$$
\omega_{a}(f, \delta)=\sup _{|t-x| \leq \delta} \sup _{x, t \in[0, a]}|f(t)-f(x)|,
$$

we denote the usual modulus of continuity of $f$ on the closed interval $[0, a]$. We know that, for a function $f \in C_{x^{2}}[0, \infty)$, the modulus of the continuity $\omega_{a}(f, \delta)$ tends to zero.

Now we give a rate of convergence theorem for the operator $P_{n, \gamma}^{\kappa}$.

Theorem 7. Let $f \in C_{x^{2}}[0, \infty)$ and $\omega_{a+1}(f, \delta)$ be its modulus of continuity on the finite interval $[0, a+1] \subset[0, \infty)$, where $a>0$. Then, for $n>\gamma$,

$$
\begin{aligned}
\left\|P_{n, \gamma}^{\kappa}(f, \cdot)-f\right\|_{C[0, a]} \leq & 6 M_{f}\left(1+a^{2}\right) \delta_{\kappa, n, \gamma}(x) \\
& +\omega_{a+1}\left(f, \sqrt{\delta_{\kappa, n, \gamma}(x)}\right) .
\end{aligned}
$$

Proof. For $x \in[0, a]$ and $t>a+1$, since $t-x>1$, we have

$$
\begin{aligned}
|f(t)-f(x)| & \leq M_{f}\left(2+x^{2}+t^{2}\right) \\
& \leq M_{f}\left(2+3 x^{2}+2(t-x)^{2}\right) \\
& \leq 6 M_{f}\left(1+a^{2}\right)(t-x)^{2} .
\end{aligned}
$$

For $x \in[0, a]$ and $t \leq a+1$, we have

$$
\begin{aligned}
|f(x)-f(t)| & \leq \omega_{a+1}(f,|t-x|) \\
& \leq\left(1+\frac{|t-x|}{\delta}\right) \omega_{a+1}(f, \delta),
\end{aligned}
$$

with $\delta>0$.

From (31) and (32) we can write

$$
\begin{aligned}
|f(t)-f(x)| \leq & 6 M_{f}\left(1+a^{2}\right)(t-x)^{2} \\
& +\left(1+\frac{|t-x|}{\delta}\right) \omega_{a+1}(f, \delta),
\end{aligned}
$$

for $x \in[0, a]$ and $t \geq 0$. Thus,

$$
\begin{aligned}
\mid P_{n, \gamma}^{\kappa} & (f, x)-f(x) \mid \\
\leq & P_{n, \gamma}^{\kappa}(|f(t)-f(x)|, x) \\
\leq & 6 M_{f}\left(1+a^{2}\right) P_{n, \gamma}^{\kappa}\left((t-x)^{2}, x\right) \\
& \quad+\omega_{a+1}(f, \delta)\left(1+\frac{1}{\delta} P_{n, \gamma}^{\kappa}\left((t-x)^{2}, x\right)^{1 / 2}\right) .
\end{aligned}
$$

Hence, by Schwarz's inequality and Lemma 3, for $x \in[0, a]$,

$$
\begin{aligned}
\left|P_{n, \gamma}^{\kappa}(f, x)-f(x)\right| & \\
\leq & 6 M_{f}\left(1+a^{2}\right) \delta_{\kappa, n, \gamma}(x) \\
& +\omega_{a+1}(f, \delta)\left(1+\frac{1}{\delta} \sqrt{\delta_{\kappa, n, \gamma}(x)}\right) .
\end{aligned}
$$

By taking $\delta=\sqrt{\delta_{\kappa, n, \gamma}(x)}$, we get

$$
\begin{aligned}
\left\|P_{n, \gamma}^{\kappa}(f, \cdot)-f\right\|_{C[0, a]} \leq & 6 M_{f}\left(1+a^{2}\right) \delta_{\kappa, n, \gamma}(x) \\
& +\omega_{a+1}\left(f, \sqrt{\delta_{\kappa, n, \gamma}(x)}\right),
\end{aligned}
$$

which proves the theorem.

Now we will discuss the weighted approximation theorem, where the approximation formula holds true on the interval $[0, \infty)$.

Theorem 8. If $f \in C_{x^{2}}^{*}[0, \infty), \lim _{n \rightarrow \infty} \kappa_{n}=0$, and $n>\gamma$, then,

$$
\lim _{n \rightarrow \infty}\left\|P_{n, \gamma}^{\kappa_{n}}(f, \cdot)-f\right\|_{x^{2}}=0 .
$$

Proof. Using the theorem in [12] we see that it is sufficient to verify the following three conditions:

$$
\lim _{n \rightarrow \infty}\left\|P_{n, \gamma}^{\kappa_{n}}\left(t^{r}, x\right)-x^{r}\right\|_{x^{2}}=0, \quad r=0,1,2 .
$$

Since $P_{n, \gamma}^{\kappa_{n}}(1, x)=1$, the first condition of (38) is fulfilled for $r=0$. By Lemma 2 we have

$$
\begin{aligned}
\left\|P_{n, \gamma}^{\kappa_{n}}(t, x)-x\right\|_{x^{2}} & =\sup _{x \in[0, \infty)} \frac{\left|P_{n, \gamma}^{\kappa_{n}}(t, x)-x\right|}{1+x^{2}} \\
& \leq\left|\frac{x}{1-\kappa_{n}}-x\right| \sup _{x \in[0, \infty)} \frac{x}{1+x^{2}} \leq \frac{\kappa_{n} x}{1-\kappa_{n}},
\end{aligned}
$$

and the second condition of (38) holds for $r=1$ as $n \rightarrow \infty$ with $\kappa_{n} \rightarrow 0$.

Similarly, we can write, for $n>\gamma$,

$$
\begin{aligned}
\left\|P_{n, \gamma}^{\kappa_{n}}\left(t^{2}, x\right)-x^{2}\right\|_{x^{2}} \\
=\sup _{x \in[0, \infty)} \frac{\left|P_{n, \gamma}^{\kappa_{n}}\left(t^{2}, x\right)-x^{2}\right|}{1+x^{2}}-1 \mid \sup _{x \in[0, \infty)} \frac{x^{2}}{1+x^{2}} \\
\leq \mid \frac{n}{\left(1-\kappa_{n}\right)^{2}(n-\gamma)} \\
\quad+\left|\frac{\left(1+\left(1-\kappa_{n}\right)^{2}\right)}{(1-\kappa)^{3}(n-\gamma)}\right| \sup _{x \in[0, \infty)} \frac{x}{1+x^{2}} \\
\leq\left|\frac{\kappa_{n}^{2}(n-\gamma)+\gamma}{\left(1-\kappa_{n}\right)^{2}(n-\gamma)}\right|+\left|\frac{\left(1+\left(1-\kappa_{n}\right)^{2}\right)}{\left(1-\kappa_{n}\right)^{3}(n-\gamma)}\right|,
\end{aligned}
$$


which implies that

$$
\lim _{n \rightarrow \infty}\left\|P_{n, \gamma}^{\kappa_{n}}\left(t^{2}, x\right)-x^{2}\right\|_{x^{2}}=0 \quad \text { with } \kappa_{n} \longrightarrow 0
$$

Thus, the proof is completed.

\section{Better Error Approximation}

In this section, we modified operator (5), in such way that the linear functions are preserved. The technique, which replaced $x$ by appropriate function, was studied for many operators, for example, Bernstein, Szász, Szász-Beta operators, and so on $[13-20]$.

We start by defining

$$
r_{\kappa}(x)=(1-\kappa) x
$$

We note that $r_{\kappa}(x) \in[0, \infty)$, for any $0 \leq \kappa<1$. By replacing $x$ by $r_{\kappa}(x)$ we give the following modification of the operators $P_{n, \gamma}^{\kappa_{n}}:$

$$
\begin{aligned}
P_{n, \gamma}^{* \kappa}(f, x)= & \sum_{i=1}^{\infty} w_{\kappa}\left(i, n r_{\kappa}(x)\right) \\
& \times \int_{0}^{\infty} b_{n, i, \gamma}(t) f(t) d t+e^{-n r_{\kappa}(x)} f(0),
\end{aligned}
$$

where

$$
w_{\kappa}\left(i, n r_{\kappa}(x)\right)=n r_{\kappa}(x)\left(n r_{\kappa}(x)+i \kappa\right)^{i-1} \frac{e^{-\left(n r_{\kappa}(x)+i \kappa\right)}}{i !}
$$

and $x \in[0, \infty), n>\gamma$; the term $b_{n, i, \gamma}(t)$ is given in (5).

Lemma 9. For $x \in[0, \infty)$ and $n>\gamma$, one has

(i) $P_{n, \gamma}^{* \kappa}(1, x)=1$,

(ii) $P_{n, \gamma}^{* \kappa}(t, x)=x$,

(iii) $P_{n, \gamma}^{* \kappa}\left(t^{2}, x\right)=n x^{2} /(n-\gamma)+\left(\left(2-2 \kappa+\kappa^{2}\right) x\right) /\left((1-\kappa)^{2}(n-\right.$ $\gamma))$.

Lemma 10. For $x \in[0, \infty), n>\gamma$, and with $\varphi_{x}=t-x$, one has

(i) $P_{n, \gamma}^{* \kappa}\left(\varphi_{x}, x\right)=0$,

(ii) $P_{n, \gamma}^{* \kappa}\left(\varphi_{x}^{2}, x\right)=x^{2} \gamma /(n-\gamma)+\left(\left(2-2 \kappa+\kappa^{2}\right) x\right) /\left((1-\kappa)^{2}(n-\right.$ $\gamma))$.

Lemma 11. For $x \in[0, \infty), n>\gamma$, one has

$$
\begin{aligned}
& P_{n, \gamma}^{* \kappa}\left(\varphi_{x}^{2}, x\right) \\
& \quad \leq \frac{2+\gamma}{(1-\kappa)^{2}(n-\gamma)}\left(x+x^{2}\right)=\tau_{\kappa, n, \gamma}(x) \quad(\text { say }) .
\end{aligned}
$$

Proof. Since $\max \left\{x, x^{2}\right\} \leq x+x^{2}$ and $(1-\kappa)^{2} \leq 1$, we have

$$
\begin{aligned}
P_{n, \gamma}^{* \kappa}\left(\varphi_{x}^{2}, x\right) & \leq \frac{\left(2-2 \kappa+\kappa^{2}\right)+\gamma(1-\kappa)^{2}}{(1-\kappa)^{2}(n-\gamma)}\left(x+x^{2}\right) \\
& =\frac{\left((1-\kappa)^{2}+1\right)+\gamma(1-\kappa)^{2}}{(1-\kappa)^{2}(n-\gamma)}\left(x+x^{2}\right) \\
& \leq \frac{2+\gamma}{(1-\kappa)^{2}(n-\gamma)}\left(x+x^{2}\right),
\end{aligned}
$$

which is required.

Theorem 12. Let $f \in C_{B}[0, \infty)$. Then for $x \in[0, \infty)$ and $n>\gamma$, one has

$$
\left|P_{n, \gamma}^{* \kappa}(f, x)-f(x)\right| \leq M \omega_{2}\left(f, \sqrt{\tau_{\kappa, n, \gamma}(x)}\right) .
$$

Proof. Let $g \in W_{\infty}^{2}$ and $x \in[0, \infty)$. Using Taylor's expansion

$$
\begin{aligned}
g(t)= & g(x)+g^{\prime}(x)(t-x) \\
& +\int_{x}^{t}(t-u) g^{\prime \prime}(u) d u, \quad t \in[0, \infty)
\end{aligned}
$$

and Lemma 10, we have

$$
P_{n, \gamma}^{* \kappa}(g, x)-g(x)=P_{n, \gamma}^{* \kappa}\left(\int_{x}^{t}(t-u) g^{\prime \prime}(u) d u\right) .
$$

Also, $\left|\int_{x}^{t}(t-u) g^{\prime \prime}(u) d u\right| \leq(t-x)^{2}\left\|g^{\prime \prime}\right\|$. Thus,

$$
\begin{aligned}
\left|P_{n, \gamma}^{* \kappa}(g, x)-g(x)\right| & \\
& \leq P_{n, \gamma}^{* \kappa}\left((t-x)^{2}, x\right)\left\|g^{\prime \prime}(x)\right\| \\
& =\frac{\left(2-2 \kappa+\kappa^{2}\right) x+x^{2} \gamma(1-\kappa)^{2}}{(1-\kappa)^{2}(n-\gamma)}\left\|g^{\prime \prime}\right\| .
\end{aligned}
$$

Since $\left|P_{n, \gamma}^{* \kappa}(f, x)\right| \leq\|f\|$,

$$
\begin{aligned}
& \left|P_{n, \gamma}^{* \kappa}(f, x)-f(x)\right| \\
& \leq\left|P_{n, \gamma}^{* \kappa}(f-g, x)-(f-g)(x)\right| \\
& \quad+\left|P_{n, \gamma}^{* \kappa}(g, x)-g(x)\right| \\
& \leq 2\|f-g\|+\frac{\left(2-2 \kappa+\kappa^{2}\right) x+x^{2} \gamma(1-\kappa)^{2}}{(1-\kappa)^{2}(n-\gamma)}\left\|g^{\prime \prime}\right\| \\
& \leq 2\|f-g\|+\tau_{\kappa, n, \gamma}(x)\left\|g^{\prime \prime}\right\| .
\end{aligned}
$$

Finally taking the infimum on right side over all $g \in W_{\infty}^{2}$, we get

$$
\left|P_{n, \gamma}^{* \kappa}(f, x)-f(x)\right| \leq K_{2}\left(f, \tau_{k, n, \gamma}\right) .
$$

In view of (18), we obtain

$$
\left|P_{n, \gamma}^{* \kappa}(f, x)-f(x)\right| \leq C \omega_{2}\left(f, \sqrt{\tau_{k, n, \gamma}}\right),
$$

which proves the theorem. 
Remark 13. We claim that the error estimation in Theorem 12 is better than that of $(20)$, provided $f \in C[0, \infty)$ and $x>0$. Indeed, in order to get this better estimation we must show that $\tau_{\kappa, n, \gamma}(x) \leq \delta_{\kappa, n, \gamma}(x)+\kappa x /(1-\kappa)$. One can obtain that

$$
\begin{aligned}
& \delta_{\kappa, n, \gamma}(x)+\frac{\kappa x}{1-\kappa} \\
& \quad \leq\left(\frac{n+2+\kappa(1-\kappa)^{2}(n-\gamma)}{(1-\kappa)^{3}(n-\gamma)}\right)\left(x+x^{2}\right) \\
& \quad \leq\left(\frac{2 n+2-\gamma}{(1-\kappa)^{3}(n-\gamma)}\right)\left(x+x^{2}\right) .
\end{aligned}
$$

Also,

$$
\begin{aligned}
\tau_{\kappa, n, \gamma}(x) & \leq \delta_{\kappa, n, \gamma}(x)+\frac{\kappa x}{1-\kappa} \\
& \Longleftrightarrow \frac{2+\gamma}{(1-\kappa)^{2}(n-\gamma)}\left(x+x^{2}\right) \\
& \leq \frac{2 n+2-\gamma}{(1-\kappa)^{3}(n-\gamma)}\left(x+x^{2}\right) \\
& \Longleftrightarrow(2+\gamma) \leq \frac{2 n+2-\gamma}{(1-\kappa)} \\
& \Longleftrightarrow 2-2 \kappa+\gamma-\gamma \kappa \leq(2 n+2-\gamma) \\
& \Longleftrightarrow 2(n-\gamma)+(2+\gamma) \kappa \geq 0,
\end{aligned}
$$

which holds true, with $n>\gamma>0$ and $\kappa>0$. Thus, $\tau_{\kappa, n, \gamma}(x) \leq$ $\delta_{\kappa, n, \gamma}(x)+\kappa x /(1-\kappa)$.

\section{Stancu Approach}

In 1968, Stancu introduced Bernstein-Stancu operator, which is a linear positive operator with two parameters $\alpha$ and $\beta$ satisfying the condition $0 \leq \alpha \leq \beta$. Inspired by the Stancutype generalization of Bernstein operator and the recent important work on several other operators are discuss in [2127], we propose following modification of the operator $P_{n, \gamma}^{\kappa}$ as

$$
\begin{aligned}
\widetilde{P}_{n, \gamma}^{\kappa, \alpha, \beta}(f, x)= & \sum_{i=1}^{\infty} w_{\kappa}(i, n x) \int_{0}^{\infty} b_{n, i, \gamma}(t) f\left(\frac{n t+\alpha}{n+\beta}\right) d t \\
& +e^{-n x} f\left(\frac{\alpha}{n+\beta}\right),
\end{aligned}
$$

where $w_{\kappa}(i, n x)$ and $b_{n, i, \gamma}(t)$ are defined in (5).

Lemma 14. For $\widetilde{P}_{n, \gamma}^{\kappa, \alpha, \beta}\left(t^{s}, x\right), s=0,1,2$, the following inequalities holds:

(i) $\widetilde{P}_{n, \gamma}^{\kappa, \alpha, \beta}(1, x)=1$,

(ii) $\widetilde{P}_{n, \gamma}^{\kappa, \alpha, \beta}(t, x)=(n x+\alpha(1-\kappa)) /((n+\beta)(1-\kappa))$,

(iii) $\widetilde{P}_{n, \gamma}^{\kappa, \alpha, \beta}\left(t^{2}, x\right)=n^{3} x^{2} /\left((n+\beta)^{2}(n-\gamma)(1-\kappa)^{2}\right)+(n x(2 n+$ $2(-n+2 \alpha(n-\gamma)) \kappa+(n-4 \alpha(n-\gamma)) \kappa^{2}+2 \alpha(n-$ $\left.\left.\gamma) \kappa^{3}\right)\right) /\left((n+\beta)^{2}(n-\gamma)(1-\kappa)^{3}\right)+\alpha^{2} /(n+\beta)^{2}$.
The proof of the above lemma can be obtained by using linearity of operators and Lemma 2.

Lemma 15. If one denotes central moments by $\Phi_{n, m, \gamma}^{\kappa, \alpha, \beta}(x)=$ $\widetilde{P}_{n, \gamma}^{\kappa, \alpha, \beta}\left((t-x)^{m}, x\right), m=1,2$, then one has

$$
\begin{aligned}
& \Phi_{n, 1, \gamma}^{\kappa, \alpha, \beta}(x)=\frac{x(n \kappa+\beta \kappa-\beta)}{(n+\beta)(1-\kappa)}+\frac{\alpha}{(n+\beta)}, \\
\Phi_{n, 2, \gamma}^{\kappa, \alpha, \beta}(x) & \left(\left(n^{3} \kappa^{2}+n^{2}\left(-2 \beta \kappa+2 \beta \kappa^{2}+\gamma\left(1-\kappa^{2}\right)\right)\right.\right. \\
+ & n \beta\left(\beta(1-\kappa)^{2}+2 \gamma \kappa(1-\kappa)\right) \\
& \left.-\beta^{2} \gamma(1-\kappa)^{2}\right) \\
& \left.\times\left((n+\beta)^{2}(n-\gamma)(1-\kappa)^{2}\right)^{-1}\right) x^{2} \\
+ & \left(\left(n^{2}\left((1-\kappa)^{2}+1+2 \alpha\left(2 \kappa^{3}-5 \kappa^{2}+4 \kappa-1\right)\right)\right)\right. \\
& \times\left((n+\beta)^{2}(n-\gamma)(1-\kappa)^{3}\right)^{-1} \\
& +(n \alpha(2 \beta-2 \gamma+(-6 \beta+8 \gamma) \kappa \\
+ & \left.\quad+(6 \beta-10 \gamma) \kappa^{2}+(-2 \beta+4 \gamma) \kappa^{3}\right) \\
& \left.\quad \times\left((n+\beta)^{2}(n-\gamma)(1-\kappa)^{3}\right)^{-1}\right) x \\
& \left.+2 \alpha \beta(1-\kappa)^{3}\right) \\
&
\end{aligned}
$$

Theorem 16. Let $\widetilde{P}_{n, \gamma}^{\kappa_{n}, \alpha, \beta}$ with $n>\gamma$ be defined in (56), where $\lim _{n \rightarrow \infty} \kappa_{n}=0$. For any compact $A \subset[0, \infty)$ and for each $f \in C_{x^{2}}^{*}[0, \infty)$, one has

$$
\lim _{n \rightarrow \infty} \widetilde{P}_{n, \gamma}^{\kappa_{n}, \alpha, \beta}(f, x)=f(x), \quad \text { uniformly in } x \in A \text {. }
$$

The proof is based on Korovkin's criterion and Lemma 14.

Theorem 17. Let $f \in C_{B}[0, \infty)$ and $n>\gamma$, one has

$$
\begin{aligned}
\left|\widetilde{P}_{n, \gamma}^{\kappa, \alpha, \beta}(f, x)-f(x)\right| \leq & \omega\left(f, \Phi_{n, 1, \gamma}^{\kappa, \alpha, \beta}(x)\right) \\
& +B \omega_{2}\left(f, \sqrt{\Phi_{n, 1, \gamma}^{\kappa, \alpha, \beta}(x)+\Phi_{n, 2, \gamma}^{\kappa, \alpha, \beta}(x)}\right)
\end{aligned}
$$

for every $0 \leq \alpha \leq \beta$ and $x \in[0, \infty)$, where $B$ is a positive constant.

The proof of Theorem 17 is just similar to Theorem 6 .

Now, we establish the Voronovskaja-type asymptotic formula for the operators $\widetilde{P}_{n, \gamma}^{\kappa, \alpha, \beta}(f, x)$. 
Theorem 18. Let $f$ be bounded and integrable on $[0, \infty)$, first and second derivatives of $f$ exist at a fixed point $x \in[0, \infty)$, and $\kappa_{n} \in(0,1)$ such that $\kappa_{n} \rightarrow 0$ as $n \rightarrow \infty$; then

$$
\begin{aligned}
& \lim _{n \rightarrow \infty} n\left(\widetilde{P}_{n, \gamma}^{\kappa_{n}, \alpha, \beta}(f, x)-f(x)\right) \\
& \quad=(\alpha-\beta x) f^{\prime}(x)+\frac{1}{2}\left(\gamma x^{2}+2(1-\alpha) x\right) f^{\prime \prime}(x) .
\end{aligned}
$$

Proof. Let $f, f^{\prime}, f^{\prime \prime} \in C_{x^{2}}^{*}[0, \infty)$ and $x \in[0, \infty)$ be fixed. By Taylor's expansion we can write

$$
\begin{aligned}
f(t)= & f(x)+f^{\prime}(x)(t-x) \\
& +\frac{1}{2} f^{\prime \prime}(x)(t-x)^{2}+r(x, t)(t-x)^{2},
\end{aligned}
$$

where $r(t, x)$ is Peano form of the remainder, $r(\cdot, x) \in$ $C_{x^{2}}^{*}[0, \infty)$, and $\lim _{t \rightarrow x} r(t, x)=0$.

Applying $\widetilde{P}_{n, \gamma}^{\kappa_{n}, \alpha, \beta}$ to the previous, we obtain

$$
\begin{aligned}
n\left[\widetilde{P}_{n, \gamma}^{\kappa_{n}, \alpha, \beta}(f, x)-f(x)\right]= & n f^{\prime}(x) \widetilde{P}_{n, \gamma}^{\kappa_{n}, \alpha, \beta}(t-x, x) \\
& +\frac{1}{2} n f^{\prime \prime}(x) \widetilde{P}_{n, \gamma}^{\kappa_{n}, \alpha, \beta}\left((t-x)^{2}, x\right) \\
& +n \widetilde{P}_{n, \gamma}^{\kappa_{n}, \alpha, \beta}\left(r(t, x)(t-x)^{2}, x\right) .
\end{aligned}
$$

By Cauchy-Schwarz's inequality, we have

$$
\begin{aligned}
& \widetilde{P}_{n, \gamma}^{\kappa_{n}, \alpha, \beta}\left(r(t, x)(t-x)^{2}, x\right) \\
& \quad \leq \sqrt{\widetilde{P}_{n, \gamma}^{\kappa_{n}, \alpha, \beta}\left(r(t, x)^{2}, x\right)} \sqrt{\widetilde{P}_{n, \gamma}^{\kappa_{n}, \alpha, \beta}\left((t-x)^{4}, x\right)} .
\end{aligned}
$$

Observe that $r^{2}(x, x)=0$ and $r^{2}(\cdot, x) \in C_{x^{2}}^{*}[0, \infty)$. Then it follows that

$$
\lim _{n \rightarrow \infty} n \widetilde{P}_{n, \gamma}^{\kappa_{n}, \alpha, \beta}\left(r(t, x)^{2}, x\right)=r^{2}(x, x)=0,
$$

uniformly with respect to $x \in[0, A]$.

Now, from (63) and (64), we obtain $\lim _{n \rightarrow \infty} n \widetilde{P}_{n, \gamma}^{\kappa_{n}, \alpha, \beta}(r(t$, $\left.x)(t-x)^{2}, x\right)=0$.

Using $\kappa_{n} \rightarrow 0$ as $n \rightarrow \infty$, we obtain

$$
\begin{gathered}
\lim _{n \rightarrow \infty} n\left(\Phi_{n, 1, \gamma}^{\kappa_{n}, \alpha, \beta}(x)\right)=\alpha-\beta x, \\
\lim _{n \rightarrow \infty} n\left(\Phi_{n, 2, \gamma}^{\kappa_{n}, \alpha, \beta}(x)\right)=\gamma x^{2}+2(1-\alpha) x .
\end{gathered}
$$

Using above limits, we have

$$
\begin{aligned}
\lim _{n \rightarrow \infty} n\left[\widetilde{P}_{n, \gamma}^{\kappa_{n}, \alpha, \beta}(f, x)-f(x)\right] \\
=\lim _{n \rightarrow \infty} n\left[f^{\prime}(x) \widetilde{P}_{n, \gamma}^{\kappa_{n}, \alpha, \beta}(t-x, x)\right. \\
\left.\quad+\frac{1}{2} f^{\prime \prime}(x) \widetilde{P}_{n, \gamma}^{\kappa_{n}, \alpha, \beta}\left((t-x)^{2}, x\right)\right] \\
\quad+\lim _{n \rightarrow \infty} n\left[\widetilde{P}_{n, \gamma}^{\kappa_{n}, \alpha, \beta}\left(r(t, x)(t-x)^{2}, x\right)\right] \\
=(\alpha-\beta x) f^{\prime}(x)+\frac{1}{2}\left(\gamma x^{2}+2(1-\alpha) x\right) f^{\prime \prime}(x),
\end{aligned}
$$

which proves the theorem.
Remark 19. In particular, if $\alpha=\beta=0$ and $\gamma=1$, then the operators $\widetilde{P}_{n, \gamma}^{\kappa_{n}, \alpha, \beta}(f, x), \kappa_{n} \in(0,1)$ such that $\kappa_{n} \rightarrow 0$ as $n \rightarrow$ $\infty$, reduce to the Jain-Beta operators recently introduced by Tarabie [7]. We obtain the following conclusion of the above asymptotic formula for the Jain-Beta operator in the ordinary approximation as follows:

$$
\lim _{n \rightarrow \infty} n\left[\widetilde{P}_{n, 1}^{\kappa_{n}, 0,0}(f, x)-f(x)\right]=\frac{1}{2}\left(x^{2}+2 x\right) f^{\prime \prime}(x) .
$$

\section{Conflict of Interests}

The authors declare that there is no conflict of interests regarding the publication of this paper.

\section{Acknowledgments}

The authors would like to express their deep gratitude to the anonymous learned referees for their valuable suggestions and comments. The second author is thankful to the Department of Mathematics, St. Xavier College, Ahmedabad, Gujarat, for carrying out his research work under the supervision of Dr. Vishnu Narayan Mishra at SVNIT, Surat, Gujarat, India. Special thanks are due to Professor Jacob C. Engwerda for kind cooperation and smooth behavior during communication and for the efforts to send the reports of the paper timely.

\section{References}

[1] P. C. Consul and G. C. Jain, "A generalization of the Poisson distribution," Technometrics, vol. 15, no. 4, pp. 791-799, 1973.

[2] G. C. Jain, "Approximation of functions by a new class of linear operators," Journal of the Australian Mathematical Society, vol. 13, pp. 271-276, 1972.

[3] G. Mirakyan, "Approximation des fonctions continues au moyen de polynômes de la forme $e^{-n x} \sum_{k=0}^{m} C_{k, n} \chi^{k}$," Doklady Akademiia Nauk SSSR, vol. 31, pp. 201-205, 1941.

[4] A. Sahai and G. Prasad, "On the rate of convergence for modified Szász-Mirakyan operators on functions of bounded variation," Institut Mathématique, vol. 53, no. 67, pp. 73-80, 1993.

[5] V. Gupta and R. P. Pant, "Rate of convergence for the modified Szász-Mirakyan operators on functions of bounded variation," Journal of Mathematical Analysis and Applications, vol. 233, no. 2, pp. 476-483, 1999.

[6] S. Umar and Q. Razi, "Approximation of function by a generalized Szasz operators," Communications de Faculté des Sciences de l'Université d'Ankara, vol. 34, pp. 45-52, 1985.

[7] S. Tarabie, "On Jain-beta linear operators," Applied Mathematics \& Information Sciences, vol. 6, no. 2, pp. 213-216, 2012.

[8] V. Gupta, G. S. Srivastava, and A. Sahai, "On simultaneous approximation by Szász-beta operators," Soochow Journal of Mathematics, vol. 21, no. 1, pp. 1-11, 1995.

[9] D. K. Dubey and V. K. Jain, "Rate of approximation for integrated Szász-Mirakyan operators," Demonstratio Mathematica, vol. 41, no. 4, pp. 879-886, 2008.

[10] P. P. Korovkin, "On convergence of linear positive operators in the space of continuous functions," Doklady Akademiia Nauk SSSR, vol. 90, pp. 961-964, 1953. 
[11] R. A. DeVore and G. G. Lorentz, Constructive Approximation, vol. 303, Springer, Berlin, Germany, 1993.

[12] A. D. Gadžiev, "Theorems of the type of P. P. Korovkin's theorems," Matematicheskie Zametki, vol. 20, no. 5, pp. 781-786, 1976.

[13] O. Duman, M. A. Özarslan, and H. Aktuğlu, "Better error estimation for Szász-Mirakjan-Beta operators," Journal of Computational Analysis and Applications, vol. 10, no. 1, pp. 53-59, 2008.

[14] O. Duman and M. A. Özarslan, "Szász-Mirakjan type operators providing a better error estimation," Applied Mathematics Letters, vol. 20, no. 12, pp. 1184-1188, 2007.

[15] H. Gonska, P. Piţul, and I. Raşa, "General King-type operators," Results in Mathematics, vol. 53, no. 3-4, pp. 279-286, 2009.

[16] O. Agratini and O. Dogru, "Weighted approximation by $q$ Szász-king type operators," Taiwanese Journal of Mathematics, vol. 14, no. 4, pp. 1283-1296, 2010.

[17] V. N. Mishra, K. Khatri, and L. N. Mishra, "Some approximation properties of q-Baskakov-Beta-Stancu type operators," Journal of Calculus of Variations, vol. 2013, Article ID 814824, 8 pages, 2013.

[18] J. P. King, "Positive linear operators which preserve $x^{2}$," Acta Mathematica Hungarica, vol. 99, no. 3, pp. 203-208, 2003.

[19] V. N. Mishra, K. Khatri, L. N. Mishra, and Deepmala, "Inverse result in simultaneous approximation by Baskakov-DurrmeyerStancu operators," Journal of Inequalities and Applications, vol. 2013, article 586, 2013.

[20] O. Agratini, "Linear operators that preserve some test functions," International Journal of Mathematics and Mathematical Sciences, vol. 2006, Article ID 94136, 11 pages, 2006.

[21] I. Büyükyazici and Ç. Atakut, "On Stancu type generalization of q-Baskakov operators," Mathematical and Computer Modelling, vol. 52, no. 5-6, pp. 752-759, 2010.

[22] D. K. Verma, V. Gupta, and P. N. Agrawal, "Some approximation properties of Baskakov-Durrmeyer-Stancu operators," Applied Mathematics and Computation, vol. 218, no. 11, pp. 6549-6556, 2012.

[23] V. N. Mishra and P. Patel, "A short note on approximation properties of Stancu generalization of $q$-Durrmeyer operators," Fixed Point Theory and Applications, vol. 2013, article 84, 5 pages, 2013.

[24] S. G. Gal, V. Gupta, D. K. Verma, and P. N. Agrawal, "Approximation by complex Baskakov-Stancu operators in compact disks," Rendiconti del Circolo Matematico di Palermo, vol. 61, no. 2, pp. 153-165, 2012.

[25] V. N. Mishra, K. Khatri, and L. N. Mishra, "On simultaneous approximation for Baskakov-Durrmeyer-Stancu type operators," Ultra Scientist of Physical Sciences, vol. 24, no. 3, pp. 567$577,2012$.

[26] V. N. Mishra, H. H. Khan, K. Khatri, and L. N. Mishra, "Hypergeometric representation for Baskakov-Durrmeyer-Stancu type operators," Bulletin of Mathematical Analysis and Applications, vol. 5, no. 3, pp. 18-26, 2013.

[27] V. N. Mishra, K. Khatri, and L. N. Mishra, "Statistical approximation by Kantorovich type discrete q-Beta operators," Advances in Difference Equations, vol. 2013, article 345, 2013. 


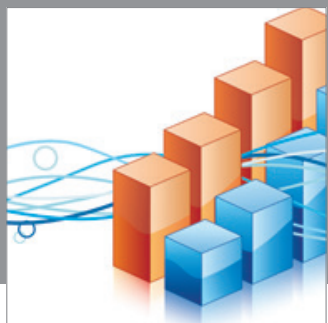

Advances in

Operations Research

mansans

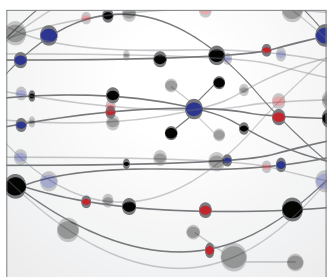

The Scientific World Journal
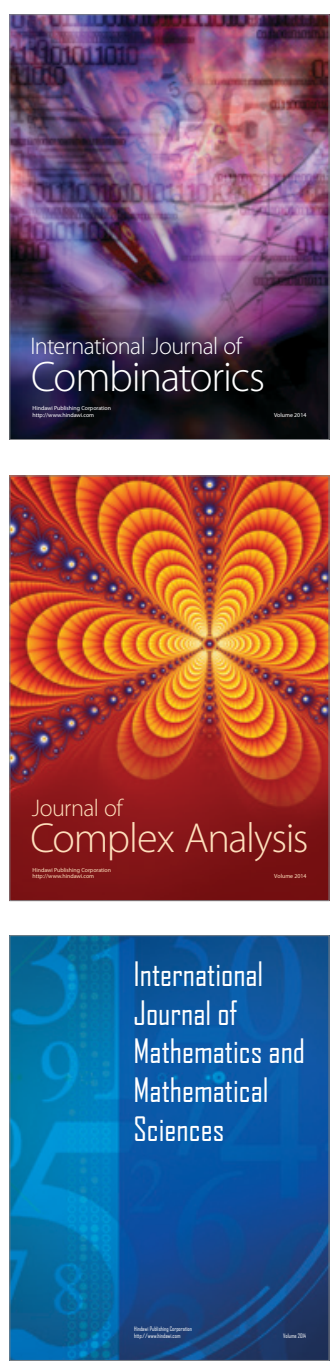
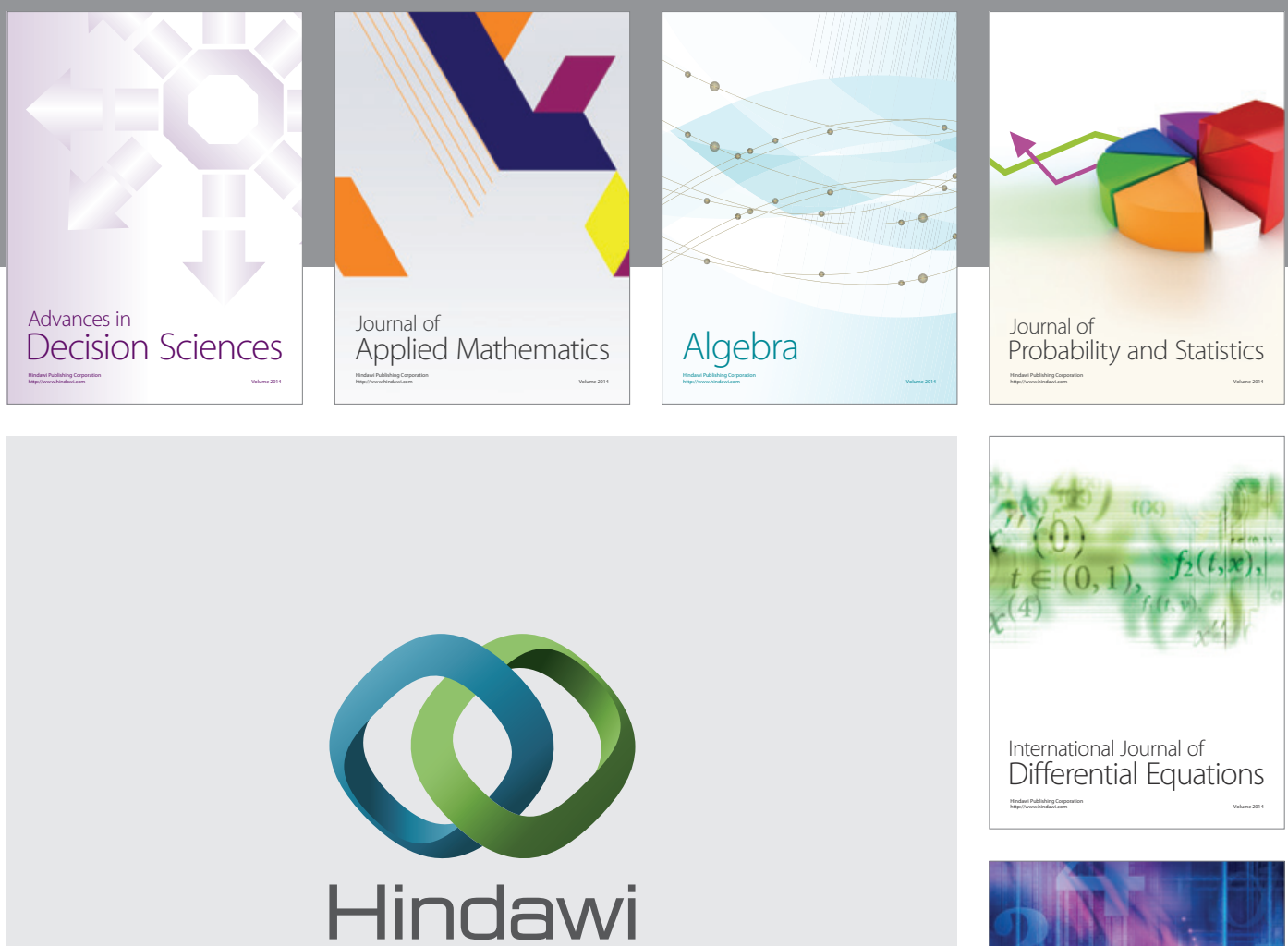

Submit your manuscripts at http://www.hindawi.com
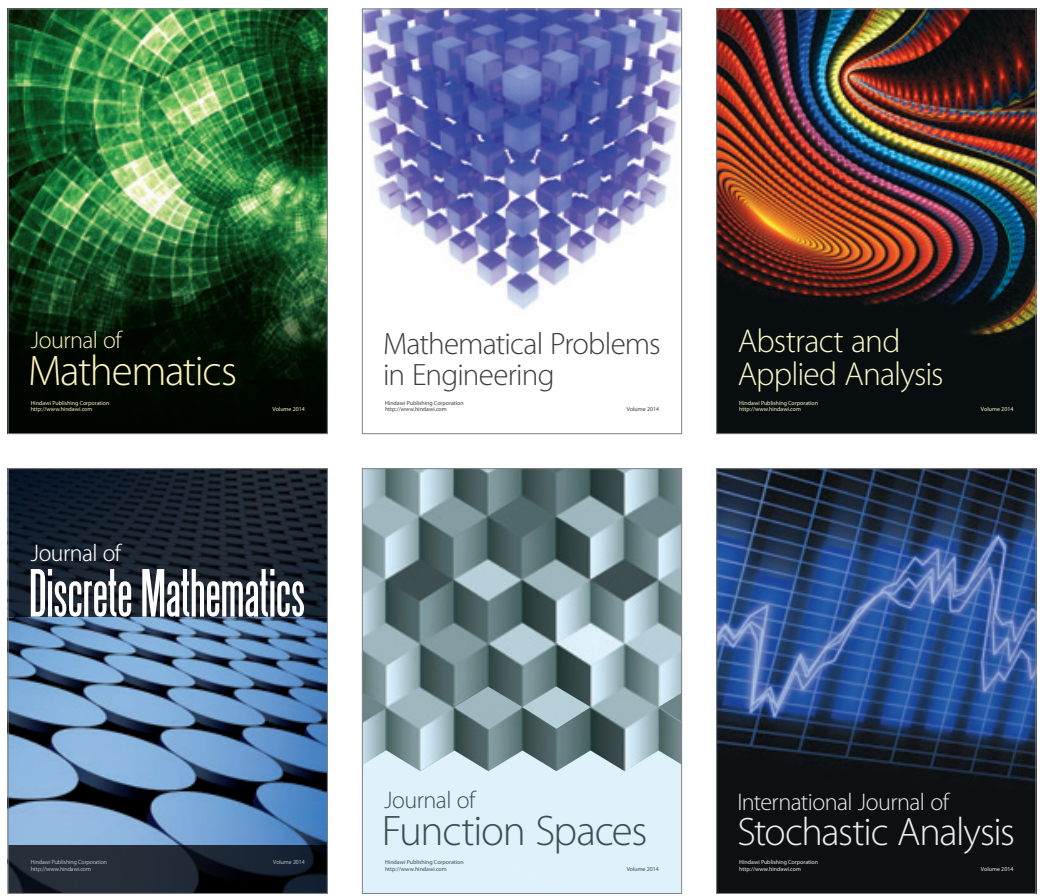

Journal of

Function Spaces

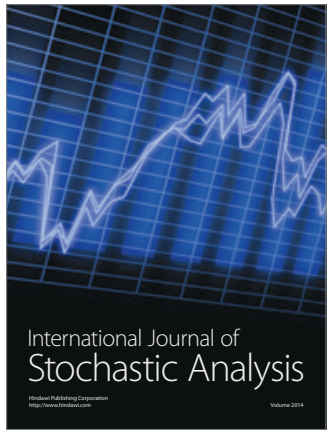

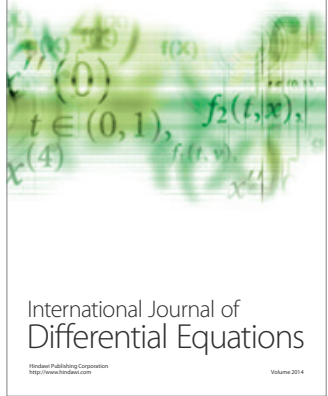
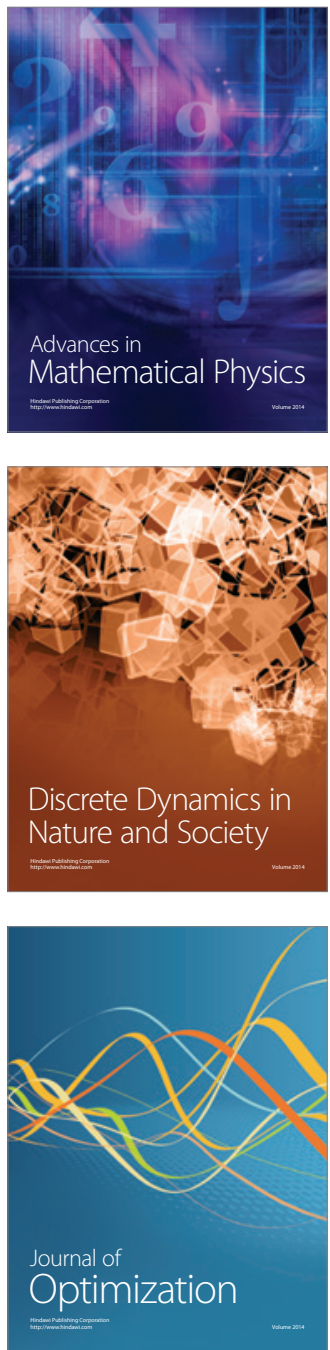